\title{
Activation and molecular recognition of the GPCR rhodopsin - Insights from time-resolved fluorescence depolarization and single molecule experiments
}

Tai-Yang Kim, Thomas Schlieter, Sebastian Haase, and Ulrike Alexiev Physics Department, Freie Universität Berlin, Arnimallee 14, D-14195 Berlin, Germany

Correspondence: Dr. Ulrike Alexiev, Physics Department, Freie Universität Berlin, Arnimallee 14, D-14195 Berlin, Germany, Phone: +49-30-838-55157, Fax: +49-30-838-56150, email: alexiev@physik.fu-berlin.de

Keywords:

GPCR, visual phototransduction, rhodopsin, GPCR helix 8, protein dynamics, time-resolved fluorescence depolarization, fluorescence anisotropy, surface potential, single particle tracking 


\begin{abstract}
The cytoplasmic surface of the G-protein coupled receptor (GPCR) rhodopsin is a key element in membrane receptor activation, molecular recognition by signaling molecules, and receptor deactivation. Understanding of the coupling between conformational changes in the intramembrane domain and the membrane-exposed surface of the photoreceptor rhodopsin is crucial for the elucidation of molecular mechanism in GPCR activation. As little is known about protein dynamics, particularly the conformational dynamics of the cytoplasmic surface elements on the nanoseconds timescale, we utilized time-resolved fluorescence anisotropy experiments and site-directed fluorescence labeling to provide information on both, conformational space and motion. We summarize our recent advances in understanding rhodopsin dynamics and function using time-resolved fluorescence depolarization and single molecule fluorescence experiments, with particular focus on the amphipathic helix 8, lying parallel to the cytoplasmic membrane surface and connecting transmembrane helix 7 with the long C-terminal tail.
\end{abstract}




\section{Introduction}

Rhodopsin is the photoreceptor involved in the signaling process that eventually converts a photon into a visual response in vertebrates (Sakmar 2002). Specifically, bovine rhodopsin consists of a polypeptide chain of 348 amino acids known as opsin, folded into a seven transmembrane (TM) $\alpha$-helical motif, the main structural GPCR signature, interconnected by hydrophilic extramembraneous loops (Fig.1). The visual receptor rhodopsin was the first GPCR with a known X-ray crystal structure (Palczewski et al. 2000; Li et al. 2004). The receptor binding site contains the chromophore 11-cis-retinal covalently bound to K296 in TM helix 7 via a protonated Schiff base linkage, having E113 in TM helix 3 as its primary counter-ion (Sakmar et al. 1989). This salt-bridge maintains the inactive conformation of the rhodopsin dark state. GPCRs share highly conserved residues (80-100\%), such as the sequence motifs D(E)RY in TM helix 3 and NPxxY in TM helix 7, and a stabilizing Cys-Cys disulfide bond (Fig.1), which all play important functional roles (Ballesteros et al. 2001; Rader et al. 2004). Thus, the large rhodopsin-like GPCR subfamily A is thought to operate through similar conserved activation mechanisms (Smith 2010; Deupi and Kobilka, 2010).

The retinal molecule in rhodopsin acts as an inverse agonist until photoactivation of the receptor by light absorption induces its isomerization to its all-trans configuration. Photoactivation results in retinal isomerization from 11-cis to all-trans on the femtosecond time scale and deprotonation of the nitrogen in the SB group in the metarhodopsin-I (MetaI) to metarhodopsin-II (MetaII) transition with internal proton transfer to E113 (Jager et al. 1994; Kukura et al. 2005; Ludeke et al. 2005; Okada et al. 2004). Proton transfer to E113 corresponds to binding of a cationic part of diffusible ligands to other rhodopsin-like receptors (Elling et al. 1999). These events initiate conformational and H-bonding changes in the receptor and proton uptake by rhodopsin that result within milliseconds in the formation of the active photoproduct MetaII, which is capable to interact via its extramembraneous cytoplasmic loops with the signaling protein transducin $\left(\mathrm{G}_{\mathrm{T}}\right)$, a member of the G-protein superfamily (Fig. 1) (Sakmar 2002). The cytoplasmic TM helix 3/TM helix 6 network (Fig. 1 and 3), including glutamic acid in position 134 (E134) of the conserved E(D)RY motif at the cytoplasmic end of TM helix 3, is supposed to be involved in proton uptake. The ERY motif was shown to act as an autonomous protonation switch depending on lipid interaction (Madathil and Fahmy 2009). Protonation of E134 in MetaII has been recently confirmed by FTIR spectroscopy (Vogel et al. 2008). The active state MetaII is formed through a complex 
cycle of a series of intermediates, including bathorhodopsin, lumirhodopsin, and MetaI, which are formed after retinal isomerization on the picosecond to millisecond time scale (Fig. 1C). These photointermediates are mainly distinguished by the spectroscopic properties (absorption maximum $\left(\lambda_{\max }\right)$ ) of their retinal absorption band. As a measure for the equilibrium between MetaII and its inactive precursor MetaI, the accompanying large spectral shift of $\lambda_{\max }($ MetaI $)=480 \mathrm{~nm}$ to $\lambda_{\max }($ MetaII $)=380 \mathrm{~nm}$ (Fig. 1E), indicative for the deprotonation of the retinal Schiff base linkage, is often used in spectroscopic studies (Kirchberg et al. 2010; Mahalingam et al 2008). The rhodopsin activation process leads to large-scale conformational changes in the protein (Farrens et al. 1996; Hubbell et al. 2003; Kim et al. 2009; Kirchberg et al. 2010; Knierim et al. 2008; Lehmann et al. 2007; Park et al. 2008; Scheerer et al. 2008), which involve helix motions and eventually trigger a cascade of biochemical reactions known as the visual phototransduction cascade (Arshavsky et al. 2002). Molecular recognition of $\mathrm{G}_{\mathrm{T}}$ by MetaII induces subunit dissociation of the heterotrimeric Gprotein, amplifying the light signal (Fig.1D). The enzymatic cascade of phototransduction can be viewed as a highly complex system of interacting functional cycles consisting of the rhodopsin (receptor) cycle, transducin (G-protein) cycle, phosphodiesterase (PDE) (effector) cycle, cyclic guanosine monophosphate (cGMP) and $\mathrm{Ca}^{2+}$ (second messenger) cycle as well as the proteins/cycles involved in deactivation (rhodopsin kinase and arrestin) and regeneration (retinoid cycle) of rhodopsin (Fig.1D) .

The macromolecules involved in phototransduction are embedded or attached to the surface of the disk membranes or plasma membrane of rod outer segments (ROS). These segments, which are highly differentiated neurons acting as photoreceptor cells, contain up to 1000-2000 distinct disk-like organelles (disk) enclosed by the plasma membrane (Fig.1A). The main protein $(\sim 90 \%)$ of the bilayered disk membranes is rhodopsin. About half of the disk membrane area is covered with rhodopsin resulting in a high protein to lipid ratio (1: 67 for bovine rhodopsin (Daemen and Bonting 1969)). The average number of rhodopsin molecules per disk is in the order of $10^{4}-10^{5}$, while the concentration of the G-protein transducin in the ROS is $\sim 500 \mu \mathrm{M}$ (Pugh and Lamb 2000). A single photon can activate up to $\sim 3000$ transducin molecules through the catalytic activity of the light-activated rhodopsin (MetaII) molecule and a single PDE molecule might activate a maximum of $\sim 2000$ cGMP molecules resulting in amplification factors of more then six orders of magnitude. Eventually, cGMP mediated ion channel closure results in hyperpolarization of the plasma membrane leading to the nerve impulse enabling vision. 
Understanding of how retinal isomerization in rhodopsin and binding of diffusible ligands in other GPCR is coupled to receptor activation requires investigation of the following questions: (1) How does the conformational change in the ligand binding pocket propagate to and manifest itself at the cytoplasmic face of the receptor? (2) What are the electrostatic changes, either vertical or horizontal to the membrane normal, associated with these conformational changes? (3) How does packing of the receptor in the membrane and its lateral diffusion affect signal transduction? In this review, we provide an update on recent advances in understanding rhodopsin dynamics and function, with particular focus on the amphipathic helix 8, lying parallel to the cytoplasmic membrane surface and connecting TM helix 7 with the long C-terminal tail.

\section{Protein dynamics at the cytoplasmic face of rhodopsin}

\section{Time-resolved fluorescence anisotropy as a tool to probe protein dynamics}

Protein motions, particularly their conformational dynamics, regulate and often constitute protein function. Dynamics and conformational changes in proteins occur on a wide range of time scales, from subpicoseconds to seconds or even longer. A large variety of experimental and theoretical techniques aim at probing internal dynamics of proteins, with a particular focus on the picosecond to microsecond time scale. For retinal proteins, such as visual rhodopsin, bacteriorhodospin, and sensory rhodopsin, various techniques comprising X-ray studies and electron crystallography on trapped intermediates, as well as time-resolved EPR, different NMR-techniques, mutagenesis studies, FTIR and fluorescence spectroscopy including time-resolved fluorescence depolarisation and molecular dynamics (MD) simulations have provided much insight into conformational changes and internal dynamics of these proteins (e.g. Alexiev et al. 2003; Brown et al. 2002; Cai et al. 1999; Crocker et al. 2006; Dunham and Farrens 1999; Dutta et al. 2010; Ernst et al. 2000; Farrens et al. 1996; Hubbell at al. 2003; Imamoto et al. 2000; Itoh et al. 2001; Kim et al. 2009; Kim et al. 2007; Klare et al. 2004; Klein-Seetharaman et al. 1999; Lehmann et al. 2007; Luecke et al. 1999; Oka et al. 1999; Radzwill et al. 2001; Sass et al. 2000; Schroder et al. 2005; Subramaniam and Henderson 2000; Vonck 2000, Ye et al. 2010, Ahuja et al.2009, Cordomi et al. 2008). 
Fluorescence spectroscopy in combination with site-directed fluorescence labeling has become an established tool to investigate the dynamics and interactions of biomolecules. In particular, time-resolved fluorescence anisotropy experiments provide information on both, conformational space and motion of a fluorophore (Munro et al. 1979; Juszczak et al. 1997). Because this probed dynamics of the dye is affected by the motion of the protein segment to which it is covalently attached, the anisotropy decay curve yields information on global and local protein dynamics as well as on the protein structure and conformational changes. Fluorescence anisotropy decay measurements are uniquely suited to obtain direct information on the local dynamics of unordered interhelical loop domains on the picosecond and nanosecond timescale. The conformational flexibility and motion of interhelical extramembraneous loops are of particular interest as they play a key role in protein-protein interactions involved in GPCR and G-protein activation, GPCR phosphorylation and deactivation. We have successfully applied this approach to the retinal proteins visual rhodopsin and bacteriorhodopsin, where we assigned an anisotropy component to a surface loop motion (Alexiev et al. 2003; Mielke et al. 2002), which allowed for the identification of protein conformational changes (Alexiev et al. 2003; Kim et al. 2009; Kirchberg et al. 2010).

The basic principle of fluorescence anisotropy relies on the excitation of the dye molecules in solution by a short (in our case 1.5 picosecond (full width half maximum)) polarized laser pulse resulting in photoselection of those dye molecules having their absorption transition dipole moments oriented along the direction of the electric field vector of the polarized exciting light (Fig. 2A inset). In Figure $2 \mathrm{~B}$ the transition dipole moment of the chromophore is indicated in the chemical structure of the dye fluorescein. After a period of time $t$, during which the dye has undergone rotational diffusion, the polarization of the emitted light is measured. Rotational diffusion changes the direction of transition dipole moments and the anisotropy measurement reveals the angular displacement of the chromophore that occurs between absorption and emission of a photon. The fluorescence anisotropy $r(t)$ at time $t$ after excitation of the fluorophore is defined as

$$
r(t)=\frac{I_{\| I}(t)-I_{\perp}(t)}{I_{I I}(t)+2 I_{\perp}(t)}
$$

where $I_{\| l}(t)$ and $I_{\perp}(t)$ are the measured parallel and perpendicular fluorescence intensities, respectively, with respect to the field vector of the exciting light pulse (Fig. 2A). The decay of 
the time-resolved anisotropy is commonly described with an exponential decay model function yielding a decay time constant or rotational correlation time $\phi$, which is determined by the rotational diffusion coefficient. The angular displacement is dependent on the rate and extent of rotational diffusion. A small fluorescent dye ( $\sim 500 \mathrm{Da})$, which tumbles free in solution depolarizes very rapidly within the time scale of the fluorescence lifetime (Fig. 2B), e.g. with a rotational correlation time of about 100-200 ps for fluorescein in aqueous solution (Alexiev et al. 2003). Larger molecules such as the purified rhodopsin molecule in detergent solution ( $\sim 40000 \mathrm{Da})$ depolarize with slower time constants, in this case with a rotational correlation time of about $50 \mathrm{~ns}$ (Mielke et al. 2002). Thus, depolarization depends on the size and shape of the rotating molecule as well as on the viscosity of the solvent. For rhodopsin disk membrane fragments, the rotational correlation time due to the tumbling of the whole membrane is on the millisecond time scale and thus far out of the fluorescence lifetime scale (about 4-10 ns) of the fluorophores used (Alexiev et al. 2003). This leads to an incomplete depolarisation on the observation timescale defined by the fluorescence lifetime of the fluorophore (Fig. 2).

When the fluorescent dye has been covalently attached to the protein (e.g. via a single cysteine residue (Alexiev et al. 2003, Moeller and Alexiev 2009)), the motional freedom of the dye, i.e. the extent of rotational diffusion, is restricted due to interactions with the surrounding protein constituents. In addition, rotational diffusion may occur not only because of the tumbling motion of the whole molecule but also because of the (faster) motion of the protein segment to which it is attached, in the case at hand to the cytoplasmic loop segments of rhodopsin. Thus, the orientational dynamics of the dye is also affected by the local flexibility of the protein. As a consequence, we will measure a time course of fluorescence depolarisation $r(t)$ for the attached fluorescent dye that exhibits a multiexponential anisotropy decay - in contrast to the simplest case of isotropic rotational diffusion of the fluorophore free in solution, where the anisotropy shows a monoexponential decay to zero (Fig. 2B). Figure $2 \mathrm{C}$ shows as an example the anisotropy decay curve of the fluorophore fluorescein covalently attached to the cysteine residue in position 316 on the cytoplasmic surface of rhodopsin in disk membranes. Two anisotropy decay components and a final anisotropy $r_{\infty}$ were observed. The fastest rotational correlation time is in the time range for the rotational diffusion of the fluorophore itself $\left(\phi_{1}=0.2 \mathrm{~ns}\right)$. The second decay time $\phi_{2}$ describes the contribution of the cytoplasmic segment motion to the rotational diffusion of the dye and its value is about $3 \mathrm{~ns}$. For comparison, a peptide with a size of about 10 amino acids would result in a rotational correlation time in the order of 1 ns (Alexiev et al. 2003). Further depolarisation on the 
timescale of the fluorescence lifetime was not observed due to the size of the membrane and the resulting slow depolarisation in milliseconds. The amplitudes $\beta$ of the decay components, which reflect the extent of the rotational diffusion for a given anisotropy decay component, can be used as a measure of the conformational space for that motion. In the simplest way, the amplitude of the loop component can be expressed as a relative value $\beta_{2}{ }^{\prime}=\beta_{2} /\left(\beta_{2}+r_{\infty}\right)$, termed relative mobility in our experiments (Alexiev et al. 2003). A small amplitude $\beta$ reflects a small conformational space and a high steric restriction by the surrounding protein constituents.

To extract real structural information from the anisotropy curves is not straightforward, however. Several empirical models have been proposed to facilitate the interpretation (Kinosta et al. 1977; Szabo 1984; Wallach 1967; Lipari and Szabo 1980). Aiming at an atomistic description, a first-principle simulation approach in direct comparison to the experimental values allowed for an extraction of the individual contributions to the depolarization and for analyzing of the dye protein interactions at atomic detail (Schroder et al. 2005). Our empirical assignment of the experimental anisotropy decay components to the individual contributions leading to depolarization (Alexiev et al. 2003) was underscored by the results from the MD simulations (Schroder et al. 2005). A common model to describe restricted rotational diffusion is the "wobbling-in-a-cone" model (Kinosta et al. 1977). In this model, the transition dipole is assumed to diffuse freely inside a cone, fixed within the molecular frame. Extension of this model for the local flexibility of protein, in our case the loop dynamics, which affect cone orientation, and possibly cone angle (Fig. 2E), leads to the "cone-in-a-cone" model (Schroder et al. 2005). Assuming that the local dye motion and the protein dynamics are uncoupled, the following equation was derived

$$
r(t)=r_{0}\left\lfloor\left(1-A_{1}\right) e^{-t / \phi_{1}}+A_{1} \rrbracket\left(1-A_{2}\right) e^{-t / \phi_{2}}+A_{2}\right\rfloor e^{-t / \phi_{G}}
$$

Here, $r_{0}$ is the initial anisotropy, $\phi_{\mathrm{G}}$ describes the isotropic overall tumbling motion of the protein-dye complex. $A_{1}$ and $A_{2}$ are the parameter describing the degree of motional restriction of the dye and the flexible protein segment, respectively. A is related to the semicone angle $\theta$ by

$$
A_{i}=\left[\frac{1}{2} \cos \theta_{i}\left(1+\cos \theta_{i}\right)\right]^{2}
$$


For isotropic diffusion with a monoexponential decay, the parameter A vanishes, while for non-isotropic diffusion, e.g. loop diffusion of rhodopsin restricted by the constituents of the protein surface, a small cone angle (high steric restriction) give rise to large values of A. Analysis of the dye and the protein motion in the simulations revealed which part and which motion of the protein is actually probed by the experiment. Furthermore, the simulations allowed to test whether the attachment of the fluorescent dye perturbs the protein dynamics or not, a prerequisite to perform these types of spectroscopic measurements. For the investigated cytoplasmic loop of the 7-helix transmembrane protein, the perturbation was quantified and found to be small (Schroder et al. 2005).

Fluorescence pump-probe measurements, where processes in the micro- and millisecond time domain (rhodopsin activation) are initiated by a nanosecond light pulse (pump flash) and monitored by time-resolved fluorescence depolarisation and lifetime measurements after a picosecond probe pulse, extend the dynamics experiments to the reaction coordinate of protein function (Kim et al. 2007). Dynamic information, as obtained from these experimental approaches, can not be obtained from x-crystallography because $\mathrm{x}$-ray structures are inherently static, whereas proteins are inherently dynamic. When applied to protein-protein interactions, which underlie the majority of biologically important signalling events in the cell, the data obtained from the time-resolved fluorescence depolarisation and fluorescence pumpprobe measurements provide unique dynamic information about how the formation process of larger signalling complexes takes place.

\section{Nanoseconds diffusional dynamics of helix 8}

Since the first x-ray crystal structure of rhodopsin (Palczewski et al. 2000) resolved the forth cytoplasmic loop between TM helix 7 and the two palmitoylated cysteines 322 and 323 as a helical structure lying parallel to the membrane surface (Fig.1, 3), it was anticipated that this helix 8 (H8) plays an important role in the GPCR signalling process due to its amphipathic properties. Located at the start of the C-terminal tail of class A GPCRs, H8 might serve as a transmitter of signalling states or is itself involved in regulated C-terminal structures. Indeed, it was shown that H8 may act as a membrane dependent conformational switch (Krishna et al. 2002). Coupling of $\mathrm{H} 8$ to the ligand binding pocket is mediated by the interhelical network (including TM helix 1, TM helix 2 and TM helix 7) that connects D83 (TM helix 2) to the conserved NPxxY motif in TM helix 7 preceding H8. In the dark (inactive) state of rhodopsin, residue F313 at the start of $\mathrm{H} 8$ is in contact with the NPxxY motif via residue Y306 (Fig. 4). 
D83 in TM helix 2 connects, via a H-bonding network, the ligand binding pocket to the NPxxY(x)F motif (Fig. 1,3).

To analyze the structural dynamics of $\mathrm{H} 8$ in the different states of the functional cycle of rhodopsin, we employed time-resolved fluorescence spectroscopy combined with site-specific fluorescence labeling of C316 in H8 (Fig. 2,3) (Alexiev et al. 2003; Kim et al. 2009; Kirchberg et al. 2010; Lehmann et al. 2007; Mielke et al. 2002). Residue 316 resides on the solvent exposed surface of $\mathrm{H} 8$ (Fig. 1B, 2D, 3B). A direct correlation of H8 conformational changes with the active receptor state MetaII was shown by FTIR/fluorescence crosscorrelation spectroscopy (Lehmann et al. 2007). The change in fluorescence intensity of certain fluorescence probes attached to $\mathrm{H} 8$ via $\mathrm{C} 316$ mirrors the equilibrium between the MetaI and MetaII state (Kim et al. 2009; Lehmann et al. 2007) and indicates environmental changes at the cytoplasmic surface of rhodopsin in the vicinity of $\mathrm{H} 8$, which are connected to the transition into the active receptor state (Fig. 4A). In combination with FTIR spectroscopy, the coupling between conformational and H-bonding changes in the intramembrane region around the retinal and the environment of H8 can be addressed (Lehmann et al. 2007). In particular, surface alterations around $\mathrm{H} 8$, monitored by intensity changes of the fluorescence reporter group at $\mathrm{H} 8$ (Fig. 4A), were shown to be highly correlated with H-bonding to Asp83 proximal of retinal Schiff base and with MetaII specific $1643 \mathrm{~cm}^{-1}$ IR absorption changes, indicative of a partial loss of secondary structure of H8 upon MetalI formation (Lehmann et al. 2007). These correlations were disrupted by limited C-terminal proteolysis but maintained upon binding of a $\mathrm{G}_{\mathrm{T}} \alpha$-subunit derived peptide, which stabilizes the activated receptor state MetaII. The results indicate that additional C-terminal tail contacts play a functionally crucial role in keeping $\mathrm{H} 8$ in a position in which its environment is strongly coupled to the retinal binding site near the Schiff base (Lehmann et al. 2007).

To provide an in-depth characterization of the internal dynamics of $\mathrm{H} 8$ as a function of the ligand-binding pocket, i.e. in the absence and presence of retinal, after light activation in the Meta-II state, as well as after phosphorylation, we systematically analyzed the time-resolved anisotropy experiments (Alexiev et al. 2003, Kim et al. 2009, Kirchberg et al. 2010). Figure 5 summarizes the contributions of $\mathrm{H} 8$ dynamics in the different states of rhodopsins functional cycle.

Our experiments revealed that in the dark state of rhodopsin the restriction of H8-diffusional motion by the constituents of the protein surface is dependent on $\mathrm{pH}$ (Alexiev et al. 2003). As this $\mathrm{pH}$ dependence coincides with the $\mathrm{pH}$-dependence of the MetaI/MetaII equilibrium (Fig. 
4), we suggested an alkaline "unlock" of H8 in the dark state possibly preventing activation of rhodopsin (Alexiev et al. 2003). A high mobility of H8 in the dark state corresponds with a shift of the MetaI/MetaII equilibrium towards the inactive precursor state MetaI (Fig. 4B).

Upon formation of MetaII, the active receptor state, the steric restriction and rotational correlation time of $\mathrm{H} 8$ diffusional motion increases, compatible with additional contacts mediated by the C-terminal tail as indicated from the FTIR experiments (Lehmann et al. 2007). The Perrin plot shown in Figure 6 clearly demonstrates the slower rotational diffusion of $\mathrm{H} 8$ in MetaII over the temperature range between 0 and $37^{\circ} \mathrm{C}$. As fluorescence depolarisation depends on size and shape of the moving segment as well as on solvent viscosity, slower H8 rotational diffusion indicates an increase in size, a shape change and/or an increase in solvent viscosity. Together with the results from FTIR measurements (Lehmann et al. 2007), it seems very likely that an increase in size, due to additional contacts with the long C-terminal tail, as well as a shape change based on partial loss of H8 secondary structure are responsible for the slower diffusion of H8 in MetaII. The former conclusion is also supported by the finding of a reduced accessibility of $\mathrm{H} 8$ from the solvent in MetaII (Mielke et al. 2002) when probed with a charged collisional quencher $\left(\mathrm{I}^{-}\right)$. Thus, our results indicated that in the active MetaII state the cytoplasmic H8 is shielded (Kirchberg et al. 2010, Mielke et al. 2002) and kept in position by additional contacts from the long C-terminal tail (Lehmann et al. 2007), which folds back toward H8 (Fig. 3). This results is important as in the X-ray crystal structures (Okada et al. 2004; Palczewski et al. 2000; Park et al. 2008; Salom et al. 2006; Scheerer et al. 2008) the long C-terminal tail is resolved only in the dark state of rhodopsin (Fig. 3).

Deactivation of rhodopsin is initiated by phosphorylation of serin and/or threonine residues at its C-terminal tail by rhodopsin kinase, followed by high-affinity binding of visual arrestin to phosphorylated light-activated rhodopsin (Gurevich and Benovic 1995; Kuhn 1982; Mendez et al. 2000). Upon light-activation of phosphorylated rhodopsin we observed only minor changes in $\mathrm{H} 8$ diffusional properties. Both, the slower rotational diffusion of $\mathrm{H} 8$ and the increased steric restriction of diffusion that are specific for active MetaII are nearly absent in phosphorylated MetaII (Kirchberg et al. 2010). This indicates that phosphorylation of the Cterminal tail probably disrupts the MetaII-specific contacts between $\mathrm{H} 8$ and the C-terminus, which subsequently lead to a de-coupling between ligand binding pocket and $\mathrm{H} 8$ and to a destabilization of the active cytoplasmic MetaII conformation. Our interpretation agrees with experimental data and simulations (Gibson et al. 2000; Hamer et al. 2003) showing partial rhodopsin (MetaII) deactivation upon rhodopsin phosphorylation. Thus, phosphorylation of 
the long C-terminal tail serves two functions, 1) allowing for high-affinity arrestin binding, and 2) decoupling of $\mathrm{H} 8$ from the intramolecular interactions with the ligand binding pocket and promoting $\mathrm{H} 8$ accessibility from the solvent. Decoupling of $\mathrm{H} 8$ dynamics might facilitate arrestin binding in a sense that it serves as a transmitter of the receptor signalling state to interacting arrestin molecules in an early encounter state.

$\mathrm{H} 8$ structure and dynamics is also sensitive to the absence of the ligand retinal in the binding pocket, i.e. the opsin state. Both in functional rhodopsin micelles and in disk membrane fragments we observed an increase in $\mathrm{H} 8$ rotational diffusion upon retinal removal (Fig. 7; Kirchberg et al. 2010). The faster rotational correlation time of H8 can be explained by a smaller size of the diffusing entity including different shape or a decrease in solvent viscosity. In either case, H8 motion is directly coupled to the change in the ligand binding pocket (Kirchberg et al. 2010). While in detergent micelles the motion of $\mathrm{H} 8$ in the retinal-devoid state is less restricted than in the rhodopsin dark state, the opposite effect was observed for opsin membranes (Fig. 7). It seems that the membrane structural organization induces contacts between H8 and cytoplasmic surface constituents, probably the long C-terminal tail, which mimics the shielding of $\mathrm{H} 8$ and the reduced conformational space indicative of the active MetaII state. It is tempting to speculate that this structural arrangement contributes to the formation of an active opsin conformation at low $\mathrm{pH}$ values in membranes (Vogel et al. 2007). The acid pH-induced active conformation of opsin was not observed in detergent micelles. These observations support also the interpretation of the opsin structure from $3 \mathrm{D}$ crystals being an activated receptor state (Park et al. 2008).

Due to its amphipathic nature, H8 dynamics is also assumed to be important for the folding pathways of GPCRs. Knowledge about the molecular folding process of mammalian membrane proteins is critical. In humans, membrane proteins constitute about one-third of all proteins (Takeda et al. 2002). Failure of a membrane protein to fold into its native threedimensional structure can impair cell functions and cause diseases (Sanders and Myers 2004), such as retinitis pigmentosa, where misfolding of rhodopsin has been implicated in contributing to disease. We could show that H8 dynamics is highly sensitive to even the slightest conformational change in the protein core, i.e. the retinal binding pocket, upon unfolding with SDS (Dutta et al. 2010a; Dutta et al. 2010b). Structural changes that involve $\mathrm{H} 8$ in the folding process may have long ranging effects due to the conserved $\mathrm{NPxxY}(\mathrm{x}) \mathrm{F}$ motif in TM helix 7 preceding cytoplasmic H8. From the 7-helix transmembrane retinal protein bacteriorhodopsin a double mutant is known, which displays long-range effects 
between the cytoplasmic end of TM helix 7 and the retinal binding pocket (Alexiev et al. 2000; Heyne et al. 2000), supporting the idea that subtle changes at this C-terminal region may affect properties of the protein core. Exchanging the third cytoplasmic loop of bacteriorhodopsin with the third intracellular loop of the aminergic $\alpha_{2 b}$-adrenergic receptor also leads to long-range effects from the cytoplasmic surface to the H-bonding networks in the extracellular part of the membrane protein (Jaakola et al. 2005), emphasizing the highly coupled intramolecular interactions that are capable of "transducing" structural perturbations (e.g. signals) across the cellular membrane. Surface charge changes may also contribute. For example, we have shown that conformational changes of membrane surface elements in bacteriorhodopsin were dependent on surface charge changes (Alexiev et al. 2003; Alexiev et al. 1995).

\section{Electrostatics at the cytoplasmic face of rhodopsin}

Using the surface potential dependence of the proton concentration near a charged membrane surface, we evaluated the electrostatic changes upon rhodopsin activation and opsin formation by a $\mathrm{pH}$-sensitive reporter group directly bound to the protein surface (Moeller and Alexiev 2009). This approach extends earlier experiments, where the partitioning of spin-labeled amphiphiles into the interfacial region of rhodopsin was used to extract information about rhodopsin surface electrostatics (Cafiso and Hubbell 1980; Hubbell 1990; Tsui et al. 1990). As the difference between surface $\mathrm{pH}$ and the $\mathrm{pH}$ value in the buffered bulk solution is related to the surface potential at a given salt concentration, the surface charge density can be calculated from the Gouy-Chapman equation (Moeller and Alexiev 2009). Summarizing our results (Moeller and Alexiev 2009), we find that the negative surface charge density in the dark state of rhodopsin is lowered to more positive values upon MetalI formation amounting to a total charge change of about three negative charges on the cytoplasmic surface including surrounding lipids. Comparison with experiments on solubilized rhodopsin in detergent micelles reveals the contribution of about two negatively charged lipids to the observed charge change, leaving about one charge as the net charge change in rhodopsin. Thus, our results clearly demonstrate that the surface charge change upon rhodopsin activation combines contributions from negatively charged lipids and the protein itself. One possibility to explain the net charge change of rhodopsins cytoplasmic surface is by the protonation of E134 in the second cytoplasmic loop. Several studies published in the past point to a protonation of this residue in MetaII (Fahmy et al. 2000; Arnis and Hofmann 1993; Arnis et al. 1994; Mahalingam et al. 2008). In addition, theoretical acid-base equilibria calculations in 
rhodopsin (Periole et al. 2004) as well as experimental evidence (Madathil and Fahmy 2009) shows that especially E134 is sensitive to its environment. Changing from a hydrophilic to a more hydrophophic environment leads to a change in the protonation state of E134 at physiological $\mathrm{pH}$ with a $K_{\mathrm{a}}$ shift from 5.3 to 8.0 (Periole et al. 2004), resulting in a positive charge change.

Further information about surface properties can be extracted from the intrinsic $\mathrm{p} K_{\mathrm{a}}$ values (i.e. $\mathrm{p} K_{\mathrm{a}}$ in the absence of electric potential) of the bound $\mathrm{pH}$-sensitive reporter group in the different states of rhodopsin. Both, in membrane suspension and in mixed rhodopsin/detergent micelles, an increase in the intrinsic $\mathrm{p} K_{\mathrm{a}}$ value of $0.2-0.3$ units upon light-activation of rhodopsin was observed. Using a thermodynamic cycle for the binding of fluorescein to the cytoplasmic surface of rhodopsin one can calculate the cost of electrostatic free energy $\Delta \Delta \mathrm{G}$ for the $\mathrm{pH}$-indicator dye to be transferred from solution to the surface of the protein. The observed increase in $\Delta \Delta \mathrm{G}$ is due to the electrostatic interactions of the $\mathrm{pH}$-indicator dye with the protein backbone and other amino acid side chains and changes in the interaction with the aqueous environment. The further increase in $\Delta \Delta \mathrm{G}$ of about $1 \mathrm{~kJ} / \mathrm{mol}$ upon light activation for both, the rhodopsin membrane suspension and rhodopsin micelles, suggests a specific surface change intimately connected with the protein surface properties in Meta II. The change in protein surface properties results from structural changes inducing a change in the electrostatic interactions of the $\mathrm{pH}$-indicator dye with the protein backbone and/or from a change of the properties of the aqueous environment at the protein/water interface. The reversal of the coupling free energy value back to the dark state level upon formation of the retinal devoid opsin state parallels the observation for the surface charge density changes (Moeller and Alexiev 2009). Both, the surface charge density of the opsin state and the coupling free energy change for the $\mathrm{pH}$-indicator dye, provide further evidence that the cytoplasmic surface properties after Meta II decay almost resemble those of the dark state (Moeller and Alexiev 2009, Klein-Seetharaman et al. 1999). This observation is in line with fluorescence pump-probe experiments revealing $\mathrm{H} 8$ environmental changes along the rhodopsin reaction coordinate (Kim et al. 2009). These experiments show the existence of multiple conformational substates within the lifetime of MetaII and a reversal back to the dark state level upon opsin formation (Fig. 5). 


\section{Surface properties, $\mathrm{H8}$ dynamics and signalling state of rhodopsin}

Combining rhodopsin surface properties (Moeller and Alexiev 2009; Kim et al. 2009; Lehmann et al. 2007) and H8 dynamics data (Alexiev et al. 2003; Kim et al. 2009; Kirchberg et al. 2010) the following picture emerges and is highlighted in Figure 5. Changes in surface properties, such as surface potential, global conformational changes and changed interactions with the aqueous environment at the cytoplasmic rhodopsin surface occur upon formation of the active receptor state MetaII. These changes are reversed upon formation of the retinaldevoid opsin state. MetaII conformation consists of a sequence of conformational substates subsequently leading to the opsin state. While proton uptake from the aqueous bulk medium parallels MetaII formation in disk membranes, kinetics of slow transient proton exchange reactions between the cytoplasmic face of rhodopsin and the aqueous bulk phase seem to correlate with the appearance of a spectrally silent conformational substate of MetaII (Kim et al. 2009). The intrinsic motion of $\mathrm{H} 8$ and its steric restriction, i.e. the diffusional dynamics on the nanosecond timescale, is distinct for the inactive dark and the light-activated MetaII state of rhodopsin with medium dynamics and medium steric restriction in the dark state and slow dynamics and high steric restriction in MetaII (Fig.5A). These receptor state specific "dynamic signatures" of $\mathrm{H} 8$ are in line with results from a mutagenesis study, where an interplay of the ligand retinal with the conserved $\mathrm{NPxxY}(\mathrm{x})_{5,6} \mathrm{~F}$ motif preceding $\mathrm{H} 8$ was shown, and which suggested that changes in the Y306-F313 interaction lead to a proper realigning of $\mathrm{H} 8$ after rhodopsin light-activation (Fritze et al. 2003). The slower dynamics of $\mathrm{H} 8$ and its higher motional restriction by the protein surface in the active receptor state MetaII as determined from our fluorescence anisotropy decay measurements qualitatively agrees with results from EPR measurements (Kusnetzov et al. 2006). These measurements show that upon light-activation the motion of the spinlabel attached to position $\mathrm{C} 316$ becomes more immobile, independent of the receptor environment (i.e. lipid bilayers or detergent micelles). This shift towards a more immobile state of the spinlabel compares to the observed increase in motional restriction of $\mathrm{H} 8$ in MetaII in our fluorescence anisotropy decay curves, which was also detected in both, the native disk membrane and detergent solubilized rhodopsin (Kim et al. 2009, Kirchberg 2010). As fluorescence anisotropy decay measurements can distinguish between the different modes of motion, i.e. the reporter group and H8 motion, our data show that indeed $\mathrm{H} 8$ motion itself is sterically restricted and $\mathrm{H} 8$ becomes more immobile.

The above discussed surface properties and their changes probably contribute to the different $\mathrm{H} 8$ rotational diffusion times observed in the dark and in the active MetaII state. Taken 
together, our data suggest a strong coupling of H8 dynamics and accessibility, cytoplasmic surface properties and intramolecular interactions with the ligand binding pocket in the dark and in the active MetaII state. However, decoupling of H8 motion from intramolecular interactions is observed upon phosphorylation of the C-terminal tail (Kirchberg et al. 2010) and is thus connected to receptor deactivation. This seems to be reversed in the opsin state (Kirchberg et al. 2010). While surface properties of the opsin state, such as surface potential, are similar to those observed in the dark state, $\mathrm{H} 8$ rotational diffusion is not. Here again, $\mathrm{H} 8$ dynamics is specific for the opsin state with fast motion compared to the dark state and the coupling to intramolecular interactions with the ligand binding pocket is restored (Kim et al. 2009, Kirchberg et al. 2010). In conclusion, our findings strongly support the role of H8 as a transmitter of GPCR signalling states and its involvement in regulated C-terminal structures.

\section{Structural organization of $\mathrm{Rh}$ in the disk membrane and impact on signaling}

The different conformational dynamics of $\mathrm{H} 8$ in the opsin state, depending on whether the molecule resides in disk membranes or is solubilized in detergent micelles, and the impact of the membrane environment on formation of an active opsin conformation at low $\mathrm{pH}$ values suggest an influence of the packing properties of rhodopsin molecules on signalling. As rhodopsin is densily packed in the disk membrane, close interactions between the molecules are very likely. The important question is whether self-association of rhodopsin, as determined by various techniques, including crosslinking, fluorescence energy transfer and modelling (Botelho et al. 2006; Dell'Orco and Schmidt 2008; Kota et al. 2006; Mansoor et al. 2006; Periole et al. 2007), is connected to the observed formation of oligomers in native rod disk membranes (Fotiadis et al. 2003). Formation of oligomers in disk membranes would have an enormous implication for phototransduction and visual signalling (Park et al. 2004; Park and Palczewski 2005; Chabre and Maire, 2005). The packing properties of rhodopsin in the disk membrane and the diffusional characteristics of molecules involved in amplification and of effector molecules will clearly affect the timing of events in the complex signal transduction cascade. Simulations of stochastic encounters between light-activated rhodopsin and $\mathrm{G}_{\mathrm{T}}$ reveal a clear dependence on rhodopsin packing and lateral diffusion of $\mathrm{G}_{\mathrm{T}}-\mathrm{a}$ respective high lateral diffusion coefficient of $\mathrm{G}_{\mathrm{T}}$ seems to be sufficient to obtain the fast responses required, even at high concentrations of immobile rhodopsin molecules (Dell'Orco and Schmidt 2008). Single molecule studies can give direct information about how individual 
molecules move and interact within the cellular context. We performed a proof-of-principleexperiment to show the feasibility of our single molecule spectroscopy approach to study the time-dependence of the light-triggered interaction between rhodopsin and transducin (Kim et al. 2009b). For this experiment we took advantage of the fact that a transducin derived peptide of the $\mathrm{G}_{\mathrm{T}} \alpha$-subunit $\mathrm{C}$-terminus recognizes the activated receptor and stabilizes the Meta-II state (Kisselev et al. 1999). As the transducin derived peptide has supposedly no affinity to dark rhodopsin (Herrmann et al. 2004) but high affinity to light-activated rhodopsin (Kisselev et al. 1999) it allows for a clear cut discrimination between the different signalling states, the dark and MetaII state. In the classical biophysical approach, the binding of transducin to lightactivated rhodopsin was monitored by kinetic light-scattering experiments (Herrmann et al. 2004). To study the interaction of individual molecules, we successfully implemented a timeresolved TIRF (total internal reflection fluorescence) microscopy assay (Kim et al 2009b) to observe the activation and biomolecular interaction kinetics of rhodopsin at the single molecule level (Fig. 8). After a short flash of light to activate rhodopsin, the interaction processes were monitored by single particle tracking (Kim et al 2009b; Kirchberg et al. 2010). Fig. $8 \mathrm{~b}$ shows an example of the single molecule binding assay. The number of spots in the image at a given time point, indicative of fluorescently labelled signaling molecules (designated $\mathrm{pG}_{\mathrm{T}} \alpha-\mathrm{F}$ in Fig. $8 \mathrm{~b}$ ) which are bound to the membrane surface, were counted and plotted as a function of time after rhodopsin activation. Without rhodopsin membranes present (control) no light induced change of the signaling molecule density was observed. These experiments also allowed to follow the lateral mobility of single activated rhodopsin molecules. Our initial results on the lateral mobility of MetaII molecules suggest confined diffusion and heterogeneous behaviour of the activated rhodopsin in the native rod disk membrane, compatible with disk membrane inhomogeneity and a semi-ordered packing of rhodopsin at a time scale, which corresponds to the millisecond activation time scale of rhodopsin (Kim et al 2009b; Kirchberg et al. 2010). Fig. 8A shows a high-resolution image of a flat-spreaded disk from bovine ROS, where transducin peptide binding highlight the rim and the indentations of the disk. Using the inherent high spatial information in single particle tracking experiments with a resolution of about $20 \mathrm{~nm}$, we constructed these so called visits maps (Fig. 8A) that allow for an identification of hotspots or places of affine binding at the rhodopsin membrane (Kirchberg et al. 2010). Using the imaging approaches described above, it is now practicable to image and analyze quantitatively the mobility, interaction pattern with signaling and regulatory molecules, as well as the oligomerization state of rhodopsin with high spatial and temporal resolution. 


\section{Acknowledgement}

Research on the dynamics of rhodopsin in the laboratory of U.A. has been funded by the

German Science Foundation, Sonderforschungsbereich 449 "Struktur und Funktion membranständiger Rezeptoren".

\section{References}

Ahuja, S., Hornak, V., Yan, E.C.Y., Syrett, N., Goncalves, J.A., Hirshfeld, A., Ziliox, M., Sakmar T.P., Sheves, M., Reeves, P.J., Smith, S.O., Eilers, M., 2009. Helix movement is coupled to displacement of the second extracellular loop in rhodopsin activation. Nat Struc \& Mol Biol 16: 168-175

Alexiev, U., Mollaaghababa, R., Khorana, H.G., Heyn, M.P., 2000. Evidence for long range allosteric interactions between the extracellular and cytoplasmic parts of bacteriorhodopsin from the mutant R82A and its second site revertant R82A/G231C. J Biol Chem 275:13431-13440

Alexiev, U., Rimke, I., Poehlmann, T., 2003. Elucidation of the nature of the conformational changes of the EF-interhelical loop in bacteriorhodopsin and of the helix VIII on the cytoplasmic surface of bovine rhodopsin: a time-resolved fluorescence depolarization study. J Mol Biol 328:705-719

Alexiev, U., Scherrer, P., Marti, T., Khorana, H.G., Heyn, M.P., 1995. Time-resolved surface charge change on the cytoplasmic side of bacteriorhodopsin. FEBS Lett 373:81-84

Arnis, S., Hofmann, K.P., 1993. Two different forms of metarhodopsin-II: Schiff base deprotonation precedes precedes proton uptake and signaling state. Proc Natl Acad Sci U S A 90: 7849-7853.

Arnis, S., Fahmy, K., Hofmann, K.P., Sakmar, T.P.,1994. A conserved carboxylic acid group mediates light-dependent proton uptake and signaling by rhoodpsin. J Biol Chem 269: 23879-23881.

Arshavsky, V.Y., Lamb, T.D., Pugh, E.N., Jr., 2002. G proteins and phototransduction. Annu Rev Physiol 64:153-87

Ballesteros, J.A., Shi, L., Javitch, J.A., 2001. Structural mimicry in G protein-coupled receptors: implications of the high-resolution structure of rhodopsin for structurefunction analysis of rhodopsin-like receptors. Mol Pharmacol 60:1-19

Botelho, A.V., Huber, T., Sakmar, T.P., Brown, M.F., 2006. Curvature and hydrophobic forces drive oligomerization and modulate activity of rhodopsin in membranes. Biophys J 91:4464-77

Brown, L.S., Needleman, R., Lanyi, J.K., 2002. Conformational change of the E-F interhelical loop in the M photointermediate of bacteriorhodopsin. J Mol Biol 317:471-478

Cafiso, D.S., Hubbell, W.L. 1980. Light-induced interfacial potential in photoreceptor membranes. Biophys.J. 30:243-263.

Cai, K., Klein-Seetharaman, J., Hwa, J., Hubbell, W.L., Khorana, H.G., 1999. Structure and function in rhodopsin: effects of disulfide cross-links in the cytoplasmic face of rhodopsin on transducin activation and phosphorylation by rhodopsin kinase. Biochemistry 38:12893-12898 
Chabre, M., le Maire, M., 2005. Monomeric G-protein-coupled receptor as a functional unit. Biochemistry 44:9395-403

Cordomi, A., Ramon, E., Garriga, P., Perez, J.J., 2008. Molecular dynamics simulation of rhodopsin point mutants at the cytoplasmic side of helices 3 and 6. J Biomol Structure \& Dynamics 25:573-587

Crocker, E., Eilers, M., Ahuja, S., Hornak, V., Hirshfeld, A., Sheves, M., Smith, S.O., 2006. Location of Trp265 in metarhodopsin II: implications for the activation mechanism of the visual receptor rhodopsin. J Mol Biol 357:163-172

Daemen, F.J., Bonting, S.L., 1969. Internal protonation in retinylidene phosphatidylethanolamine and the red-shift in rhodopsin. Nature 222:879-81

Dell'Orco, D., Schmidt, H., 2008. Mesoscopic Monte Carlo simulations of stochastic encounters between photoactivated rhodopsin and transducin in disc membranes. $\mathrm{J}$ Phys Chem B 112:4419-26

Deupi, X. \& Kobilka, B.K., 2010. Energy landscapes as a tool to integrate GPCR structure, dynamics, and function. Physiology 25: 293-303

Dunham, T.D., Farrens, D.L., 1999. Conformational changes in rhodopsin. Movement of helix F detected by site-specific chemical labeling and fluorescence spectroscopy. $\mathrm{J}$ Biol Chem 274:1683-1690

Dutta, A., Kim, T.Y., Moeller, M., Wu, J., Alexiev, U., Klein-Seetharaman, J., 2010a. Characterization of membrane protein non-native states. 2. The SDS-unfolded states of rhodopsin. Biochemistry 49:6329-6340

Dutta, A., Tirupula, K.C., Alexiev, U., Klein-Seetharaman, J., 2010b. Characterization of membrane protein non-native states. 1. Extent of unfolding and aggregation of rhodopsin in the presence of chemical denaturants. Biochemistry 49:6317-6328

Elling, C.E., Thirstrup, K., Holst, B., Schwartz, T.W., 1999. Conversion of agonist site to metal-ion chelator site in the beta(2)-adrenergic receptor. Proc Natl Acad Sci U S A 96:12322-7

Ernst, O.P., Meyer, C.K., Marin, E.P., Henklein, P., Fu, W.Y., Sakmar, T.P., Hofmann, K.P., 2000. Mutation of the fourth cytoplasmic loop of rhodopsin affects binding of transducin and peptides derived from the carboxyl-terminal sequences of transducin alpha and gamma subunits. J Biol Chem 275:1937-1943

Fahmy, K., Sakmar, T., Siebert, F., 2000. Transducin dependent protonation of glutamic acid 134 in rhodopsin. Biochemistry 39: 10607-10612

Farrens, D.L., Altenbach, C., Yang, K., Hubbell, W.L., Khorana, H.G., 1996. Requirement of rigid-body motion of transmembrane helices for light activation of rhodopsin. Science 274:768-70

Fotiadis, D., Liang, Y., Filipek, S., Saperstein, D.A., Engel, A., Palczewski, K., 2003. Atomic-force microscopy: Rhodopsin dimers in native disc membranes. Nature 421:127-128

Fritze, O., Filipek, S., Kuksa, V., Palczewski, K., Hofmann, K.P., Ernst O.P., 2003.Role of the conserved $\mathrm{NPxxY}(\mathrm{x})_{5,6} \mathrm{~F}$ motif in the rhodopsin ground state and during activation. Proc Natl Acad Sci U S A 100:2290-2295

Gibson, S.K., Parkes, J.H., Liebman, P.A., 2000. Phosphorylation modulates the affinity of light-activated rhodopsin for $\mathrm{G}$ protein and arrestin. Biochemistry 39:5738-49

Gurevich, V.V., Benovic, J.L., 1995. Visual arrestin binding to rhodopsin. Diverse functional roles of positively charged residues within the phosphorylation-recognition region of arrestin. J Biol Chem 270:6010-6

Hamer, R.D., Nicholas, S.C., Tranchina, D., Liebman, P.A., Lamb, T.D., 2003. Multiple steps of phosphorylation of activated rhodopsin can account for the reproducibility of vertebrate rod single-photon responses. J Gen Physiol 122:419-44 
Herrmann, R., Heck, M., Henklein, P., Henklein, P., Kleuss, C., Hofmann, K.P., Ernst, O.P., 2004. Sequence of Interactions in Receptor-G Protein Coupling. J Biol Chem 279: 24283-24290

Heyne, K., Herbst, J., Dominguez-Herradon, B., Alexiev, U., Diller, R., 2000. Reaction control in bacteriorhodopsin: impact of Arg82 and Asp85 on the fast retinal isomerization, studied in the second site revertant Arg82Ala/Gly231Cys and various purple and blue forms of bacteriorhodopsin. J. Phys. Chem. B 104:6053-6058

Hubbell, W.L.,1990. Transbilayer coupling mechanism for the formation of lipid asymmetry in biological membranes. application to the photoreceptor disk membrane. Biophys $\mathrm{J}$ 57: $99-108$

Hubbell, W.L., Altenbach, C., Hubbell, C.M., Khorana, H.G., 2003. Rhodopsin structure, dynamics, and activation: a perspective from crystallography, site-directed spin labeling, sulfhydryl reactivity, and disulfide cross-linking. Adv Protein Chem 63:24390

Imamoto, Y., Kataoka, M., Tokunaga, F., Palczewski, K., 2000. Light-induced conformational changes of rhodopsin probed by fluorescent alexa594 immobilized on the cytoplasmic surface. Biochemistry 39:15225-15233

Itoh, Y., Cai, K., Khorana, H.G., 2001. Mapping of contact sites in complex formation between light-activated rhodopsin and transducin by covalent crosslinking: use of a chemically preactivated reagent. Proc Natl Acad Sci U S A 98:4883-4887

Jaakola, V.P., Rehn, M., Moeller, M., Alexiev, U., Goldman, A., Turner, G.J., 2005. Gprotein-coupled receptor domain overexpression in Halobacterium salinarum: longrange transmembrane interactions in heptahelical membrane proteins. Proteins 60:41223

Jager, F., Fahmy, K., Sakmar, T.P., Siebert, F., 1994. Identification of glutamic acid 113 as the Schiff base proton acceptor in the metarhodopsin II photointermediate of rhodopsin. Biochemistry 33:10878-82

Juszczak, L.J. Zhang, Z.Y. Wu, D., Gottfried, D.S., Eads, D.D., 1997. Rapid loop dynamics of Yersinia protein tyrosine phosphatases. Biochemistry 36: 2227-2236

Kim, T.Y., Moeller, M., Winkler, K., Kirchberg, K., Alexiev, U., 2009a. Dissection of Environmental Changes at the Cytoplasmic Surface of Light-activated Bacteriorhodopsin and Visual Rhodopsin: Sequence of Spectrally Silent Steps. Photochem Photobiol 85:570-7

Kim, T.Y., Uji-i, H., Moeller, M., Muls, B., Hofkens, J., Alexiev, U., 2009b. Monitoring the interaction of a single G-protein key binding site with rhodopsin disk membranes upon light activation. Biochemistry 48:3801-3803

Kim, T.Y., Winkler, K., Alexiev, U., 2007. Picosecond multidimensional fluorescence spectroscopy: a tool to measure real-time protein dynamics during function. Photochem Photobiol 83:378-84

Kinosta, K., Kawato, S. , Ikegami, A., 1977. A theory of fluorescence polarization decay in membranes. Biophys. J. 20:289-305

Kirchberg, K., Kim, T.Y., Haase, S., Alexiev, U., 2010. Functional interaction structures of the photochromic retinal protein rhodopsin. Photochem Photobiol Sci 9:226-33

Kisselev, O.G., Meyer, C.K., Heck, M., ERnst, O.P., Hofmann, K.P., 1999. Signal transfer from rhodopsin to the G-protein: Evidence for a two-site sequential fit mechanis. Proc Natl Acad Sci U S A 96: 4898-4903

Klare, J.P., Bordignon, E., Engelhard, M., Steinhoff, H.J., 2004. Sensory rhodopsin II and bacteriorhodopsin: light activated helix F movement. Photochem Photobiol Sci 3:543547

Klein-Seetharaman, J., Getmanova, E.V., Loewen, M.C., Reeves, P.J., Khorana, H.G., 1999. NMR spectroscopy in studies of light-induced structural changes in mammalian 
rhodopsin: applicability of solution (19)F NMR. Proc Natl Acad Sci U S A 96:1374413749

Knierim, B., Hofmann, K.P., Gartner, W., Hubbell, W.L., Ernst, O.P., 2008. Rhodopsin and 9-demethyl-retinal analog: effect of a partial agonist on displacement of transmembrane helix 6 in class A G protein-coupled receptors. J Biol Chem 283:496774

Kota, P., Reeves, P.J., Rajbhandary, U.L., Khorana, H.G., 2006. Opsin is present as dimers in COS1 cells: identification of amino acids at the dimeric interface. Proc Natl Acad Sci U S A 103:3054-9

Krishna, A.G., Menon, S.T., Terry, T.J., Sakmar, T.P., 2002. Evidence that helix 8 of rhodopsin acts as a membrane-dependent conformational switch. Biochemistry 41:8298-309

Kuhn, H., 1982. Light-regulated binding of proteins to photoreceptor membranes and its use for the purification of several rod cell proteins. Methods Enzymol 81:556-64

Kukura, P., McCamant, D.W., Yoon, S., Wandschneider, D.B., Mathies, R.A., 2005. Structural observation of the primary isomerization in vision with femtosecondstimulated Raman. Science 310:1006-9

Kusnetzov, A.K., Altenbach, C., Hubbell, W., 2006. Conformational states and dynamics of rhodopsin in micelles and bilayers. Biochemistry 45:5538-50

Lehmann, N., Alexiev, U., Fahmy, K., 2007. Linkage between the intramembrane H-bond network around aspartic acid 83 and the cytosolic environment of helix 8 in photoactivated rhodopsin. J Mol Biol 366:1129-41

Li, J., Edwards, P.C., Burghammer, M., Villa, C., Schertler, G.F., 2004. Structure of bovine rhodopsin in a trigonal crystal form. J Mol Biol 343: 1409-1438.

Lipari, G., and Szabo,A.,1980. Effect of librational motion on fluorescence depolarization and nuclear magnetic resonance relaxation macromolecules and membranes. Biophys. J. 30:489-506.

Ludeke, S., Beck, M., Yan, E.C., Sakmar, T.P., Siebert, F., Vogel, R., 2005. The role of Glu181 in the photoactivation of rhodopsin. J Mol Biol 353:345-56

Luecke, H., Schobert, B., Richter, H.T., Cartailler, J.P., Lanyi, J.K., 1999. Structural changes in bacteriorhodopsin during ion transport at 2 angstrom resolution. Science 286:25561

Madathil, S., Fahmy, K., 2009. Lipid protein interactions couple protonation to conformation in a conserved cytosolic domain of $\mathrm{G}$ protein-coupled receptors. J Biol Chem 284:28801-9

Mahalingam, M., Martinez-Mayorga K, Brown MF, Vogel R., 2008 Two protonation switches control rhodopsin activation in membranes. Proc Natl Acad Sci U S A 105: 17795-17800.

Mansoor, S.E., Palczewski, K., Farrens, D.L., 2006. Rhodopsin self-associates in asolectin liposomes. Proc Natl Acad Sci U S A 103: 3060-65

Mendez, A., Burns, M.E., Roca, A., Lem, J., Wu, L.W., Simon, M.I., Baylor, D.A., Chen, J., 2000. Rapid and reproducible deactivation of rhodopsin requires multiple phosphorylation sites. Neuron 28:153-64

Mielke, T., Alexiev, U., Glasel, M., Otto, H., Heyn, M.P., 2002. Light-induced changes in the structure and accessibility of the cytoplasmic loops of rhodopsin in the activated MII state. Biochemistry 41:7875-84

Moeller, M., Alexiev, U., 2009. Surface charge changes upon formation of the signaling state in visual rhodopsin. Photochem Photobiol 85:501-508

Munro, I., Pecht, I., Stryer, L., 1979. Subnanosecond motions of tryptophan residues in proteins. Proc Natl Acad Sci U S A 76:56-60 
Oka, T., Kamikubo, H., Tokunaga, F., Lanyi, J.K., Needleman, R., Kataoka, M., 1999. Conformational change of helix $\mathrm{G}$ in the bacteriorhodopsin photocycle: investigation with heavy atom labeling and x-ray diffraction. Biophys J 76:1018-1023

Okada, T., Sugihara, M., Bondar, A.N., Elstner, M., Entel, P., Buss, V., 2004. The retinal conformation and its environment in rhodopsin in light of a new 2.2 A crystal structure. J Mol Biol 342:571-83

Palczewski, K., Kumasaka, T., Hori, T., Behnke, C.A., Motoshima, H., Fox, B.A., Le Trong, I., Teller, D.C., Okada, T., Stenkamp, R.E., Yamamoto, M., Miyano, M., 2000. Crystal structure of rhodopsin: A G protein-coupled receptor. Science 289:739-45

Park, J.H., Scheerer, P., Hofmann, K.P., Choe, H.W., Ernst, O.P., 2008. Crystal structure of the ligand-free G-protein-coupled receptor opsin. Nature 454:183-7

Park, P.S., Filipek, S., Wells, J.W., Palczewski, K., 2004. Oligomerization of G proteincoupled receptors: past, present, and future. Biochemistry 43:15643-56

Park, P.S., Palczewski, K., 2005. Diversifying the repertoire of G protein-coupled receptors through oligomerization. Proc Natl Acad Sci U S A 102:8793-4

Periole, X., Ceruso, M.A., Mehler, E.L., 2004. Acid-base equilibria in rhodopsin: dependence of the protonation state of Glu134 on its environment. Biochemsitry 43: 6858-64.

Periole, X., Huber, T., Marrink, S.J., Sakmar, T.P., 2007. G protein-coupled receptors selfassemble in dynamics simulations of model bilayers. J Am Chem Soc 129:10126-32

Pugh, E.N.J., Lamb, T.D. (2000) Phototransduction in vertebrate rods and cones: molecular mechanisms of amplification, recovery and light adaptation. Handbook of Biological Physics, 3: 183-255, Elsevier Science BV.

Rader, A.J., Anderson, G., Isin, B., Khorana, H.G., Bahar, I., Klein-Seetharaman, J., 2004. Identification of core amino acids stabilizing rhodopsin. Proc Natl Acad Sci U S A 101:7246-51

Radzwill, N., Gerwert, K., Steinhoff, H.J., 2001. Time-resolved detection of transient movement of helices $\mathrm{F}$ and $\mathrm{G}$ in doubly spin-labeled bacteriorhodopsin. Biophys J 80:2856-2866

Sakmar, T.P., 2002. Structure of rhodopsin and the superfamily of seven-helical receptors: the same and not the same. Curr Opin Cell Biol 14:189-95

Sakmar, T.P., Franke, R.R., Khorana, H.G., 1989. Glutamic acid-113 serves as the retinylidene Schiff base counterion in bovine rhodopsin. Proc Natl Acad Sci U S A 86:8309-13

Salom, D., Lodowski, D.T., Stenkamp, R.E., Le Trong, I., Golczak, M., Jastrzebska, B., Harris, T., Ballesteros, J.A., Palczewski, K., 2006. Crystal structure of a photoactivated deprotonated intermediate of rhodopsin. Proc Natl Acad Sci U S A 103:16123-8

Sanders, C.R., Myers, J.K., 2004. Disease-related misassembly of membrane proteins. Annu Rev Biophys Biomol Struct 33:25-51

Sass, H.J., Bueldt, G., Gessenich, R., Hehn, D., Neff, D., Schlesinger, R., Berendzen, J., Ormos, P., 2000. Structural alterations for proton translocation in the M state of wildtype bacteriorhodopsin. Nature 406:649-653

Scheerer, P., Park, J.H., Hildebrand, P.W., Kim, Y.J., Krauss, N., Choe, H.W., Hofmann, K.P., Ernst, O.P., 2008. Crystal structure of opsin in its G-protein-interacting conformation. Nature 455:497-502

Schroder, G.F., Alexiev, U., Grubmuller, H., 2005. Simulation of fluorescence anisotropy experiments: probing protein dynamics. Biophys J 89:3757-70

Subramaniam, S., Henderson, R., 2000. Molecular mechanism of vectorial proton translocation by bacteriorhodopsin. Nature 406:653-657

Smith, S.O.,2010. Structure and activation of the visual pigment rhodopsin. Ann Rev Biophys 39:309-28 
Szabo, A., 1984. Theory of fluorescence depolarization in macromolecules and membranes. J. Chem. Phys. 81:150-167

Takeda, S., Kadowaki, S., Haga, T., Takaesu, H., Mitaku, S., 2002. Identification of G protein-coupled receptor genes from the human genome sequence. FEBS Lett 520:97101

Tsui, F.C., Sundberg, S.A., Hubbell, W.L., 1990. Distribution of charge on photoreceptor disc membarnes and implications for charged lipid assymmetry. Biophys. J. 57: 85-97

Vogel, R., Mahalingam, M., Ludeke, S., Huber, T., Siebert, F., Sakmar, T.P., 2008. Functional role of the "ionic lock"--an interhelical hydrogen-bond network in family A heptahelical receptors. J Mol Biol 380:648-55

Vogel, R., Sakmar, T.P., Sheves, M., Siebert, F., 2007. Coupling of protonation switches during rhodopsin activation. Photochem Photobiol 83:286-92

Vonck, J., 2000. Structure of the bacteriorhodopsin mutant F219L N intermediate revealed by electron crystallography. EMBO J 19:2152-2160

Wallach, D. 1967.Effect of internal rotation on angular correlation functions. J. Chem. Phys. 47:5258-5268

Ye, S., Zaiteva, E., Caltabiano, G., Schertler, G.F.X., Sakmar, T.P., Deupi, X., Vogel, R., 2010. Tracking G-protein coupled receptor activation using genetically encoded infrared probes. Nature 464:1386-1390. 


\section{Figure legends}

\section{Figure 1}

Models of rhodopsin and the visual phototransduction cascade.

(A) Schematic of a rod cell. A disk in the rod outer segment (ROS) is high lighted in red. (B) Molecular model of rhodopsin based on the x-ray structural model of the inactive dark state (pbd-file 1U19 (Okada et al., 2004)). Amino acids belonging to the different interhelical networks as described in the text are color coded in orange, magenta, blue and green. The chromophore retinal (Ret) is colored in black. Possible phosphorylation sites (serine 334, serine 338, serine 343) in the C-terminal (C-term) tail are shown as blue spheres. The cysteine 316 in H8 (red colored), which is used as a binding site for the fluorescence label is marked by a red sphere. (C) Photochemical cycle of rhodopsin. (D) Model of the phototransduction cascade. Transducin $\left(\mathrm{G}_{\mathrm{T}}\right)$ and phosphodiesterase (PDE) activation result in cGMP mediated ion channel closure facilitating hyperpolarization of the plasma membrane and leading to the nerve impulse enabling vision. To terminate the signaling process, visual arrestin (Arr) binds to the cytosolic face of rhodopsin, thereby hindering G-protein binding and quenching metarhodopsin-II (MII) activity. Phosphorylation of rhodopsins's serins and/or threonines at its C-terminal tail by rhodopsin kinase (RK) is a prerequisite for visual arrestin binding. (E) Absorption spectra of rhodopsin in its inactive dark (black) and active MetaII (red) state.

Further abbreviations used: Rho-rhodopsin, $\mathrm{G}_{\mathrm{T}} \alpha \beta \gamma$-heterotrimeric G-protein transducin, PDE*-activated PDE, GTP-Guanosine-5'-triphosphate, GDP- guanosine-5'-diphosphate, GMP- guanosine-5'-monophosphate, cGMP-cyclic guanosine monophosphate, P-phosphate

\section{Figure 2}

Polarized time-resolved fluorescence decays.

(A) Time courses of the fluorescence intensities $I_{I I}(t)$ (red) and $I_{\perp}(t)$ (black) of fluorescein at $37^{\circ} \mathrm{C}$ in buffer $\mathrm{pH}$ 7. The fluorescence lifetime was $3.8 \mathrm{~ns}$. Inset: Scheme of measurement principle. (B) Corresponding fluorescence anisotropy decay. The inset shows the chemical structure of iodoacteamidofluorescein, the arrow indicates the transition dipole moment of the chromophore. (C) Fluorescence anisotropy decay of fluorescein covalently coupled to Cys316 at the cytoplasmic surface of rhodospin disk membranes at $37^{\circ} \mathrm{C}, 150 \mathrm{mM} \mathrm{NaCl}$, buffer $\mathrm{pH} 7$. (D) Structural model of helix 8 (red) with fluorescein (green) covalently bound to Cys 316. Surrounding transmembrane helices are shown in gray. (E) "Cone-in-a-cone" model. The schematic visualizes how protein dynamics is described by the protein segment cone (here $\mathrm{H} 8$, red)), within which the dye cone (green) freely diffuses (Alexiev et al. 2003; Schroder et al. 2005). Color coding corresponds to the colors used in (C) and (D).

Figure 3

Close up of the molecular model of rhodopsin based on the x-ray structure of the inactive dark state (pbd-file 1 U19 (Okada et al., 2004)). (A) Side view of the cytoplasmic part of rhodopsin. The amino acids belonging to the ERY and NPxxY motif and interacting residues as well as the hydrophobic residues of $\mathrm{H} 8$ are indicated. (B) Top view of the cytoplasmic surface. Possible phosphorylation sites (serine 334, serine 338, serine 343) in the C-terminal (C-ter) tail are shown as blue spheres. 


\section{Figure 4}

$\mathrm{pH}$-dependence of $\mathrm{H} 8$ conformational dynamics. (A) The fluorescence intensity ratio of the covalently bound dye measured before and after rhodopsin light activation parallels the $\mathrm{pH}$ dependence of the MetaI/MetaII equilibrium. (B) H8 mobility changes expressed as semicone angles (eq.2,3) as a function of $\mathrm{pH}$ (data from Alexiev et al. 2003; Kim et al. 2009).

\section{Figure 5}

Schematic presentation of the role of $\mathrm{H} 8$ in the functional cycle of rhodopsin. Results from time-resolved fluorescence depolarization decay, fluorescence pump-probe, and surface potential measurements are combined (A) and the derived model is presented in (B). The proposed coupling between properties of the ligand binding pocket and $\mathrm{H} 8$ dynamics is indicated by a dotted red line in (B).

\section{Figure 6}

Perrin plot of $\mathrm{H} 8$ dynamics. The rotational diffusion time constant of H8 in the inactive dark state (black) and in the MetaII state (red) at pH 6 are displayed (data from Alexiev et al 2003; Kim et al. 2009).

Figure 7

Fluorescence anisotropy decay of fluorescein covalently coupled to Cys 316 at the cytoplasmic surface of opsin disk membranes at $37^{\circ} \mathrm{C}, 150 \mathrm{mM} \mathrm{NaCl}$, buffer $\mathrm{pH} 7.5$ and opsin micelles at $20^{\circ} \mathrm{C}, 130 \mathrm{mM} \mathrm{NaCl}$, buffer $\mathrm{pH} 7.5$ (Kirchberg et al. 2010) in comparison to the respective anisotropy decay in the inactive dark state. The rotational correlation times of $\mathrm{H} 8$ are indicated.

\section{Figure 8}

Single molecule TIRF experiments of rhodopsin disk membranes.

(A) "Transducin visits maps" highlight the interaction with rhodopsin disk membranes and were constructed from the single particle traces (Kirchberg et al. 2010). The shape and membrane structure of the disk is clearly visible. (B) Kinetic single molecule binding assay for signaling molecules, here with $\mathrm{pGT} \alpha-\mathrm{F}$ (red trace), a fluorescently labeled transducin derived peptide, which stabilizes MetaII (Kim et al. 2009b). For comparison, the time trace of MetaII formation ( $\triangle \mathrm{A}$ at $380 \mathrm{~nm}$ ) is shown. The schematic shows individual fluorescently labelled molecules (white dots) interacting with rhodopsin containing disk membranes. 


\section{Figure 1}
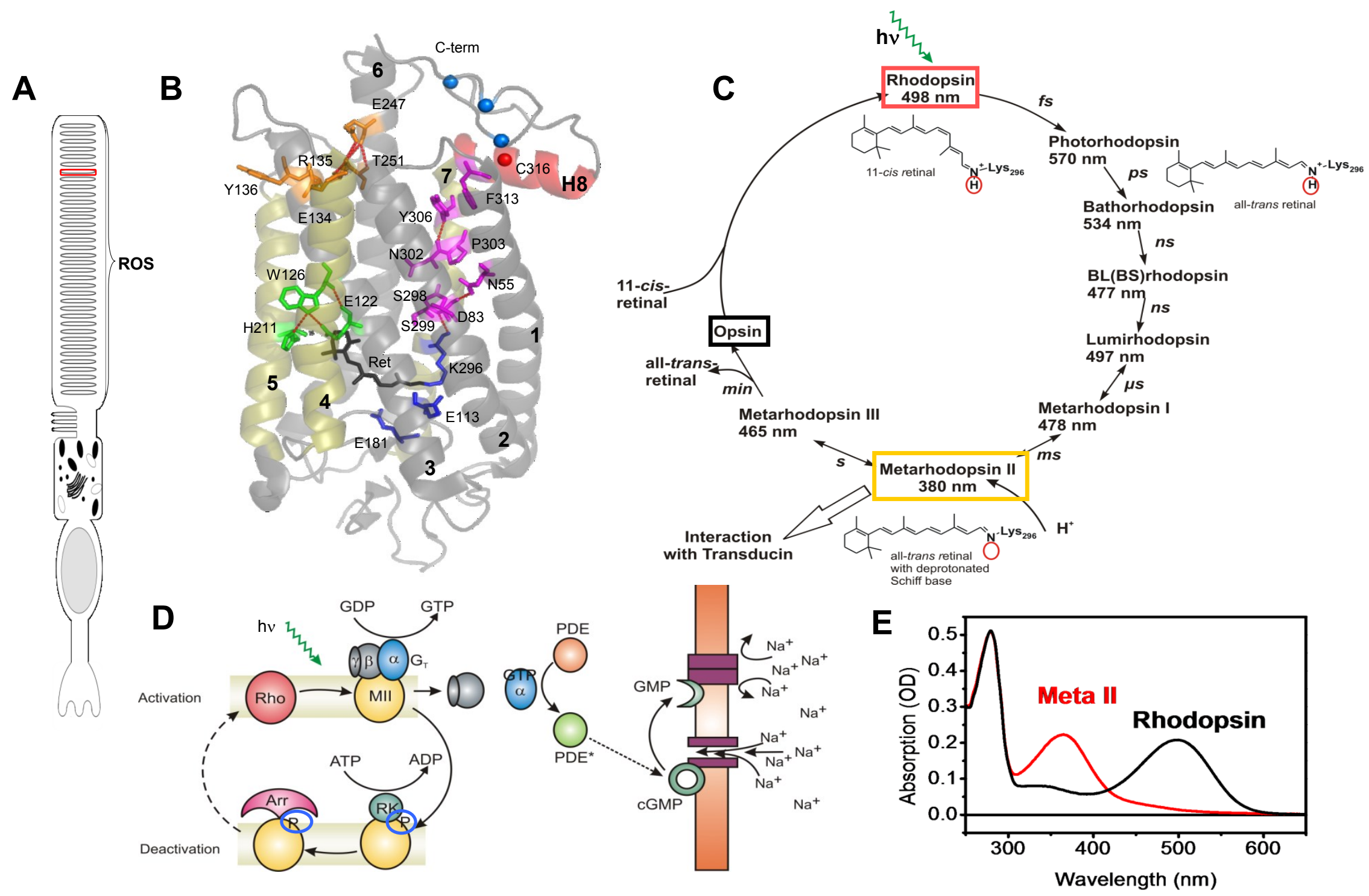

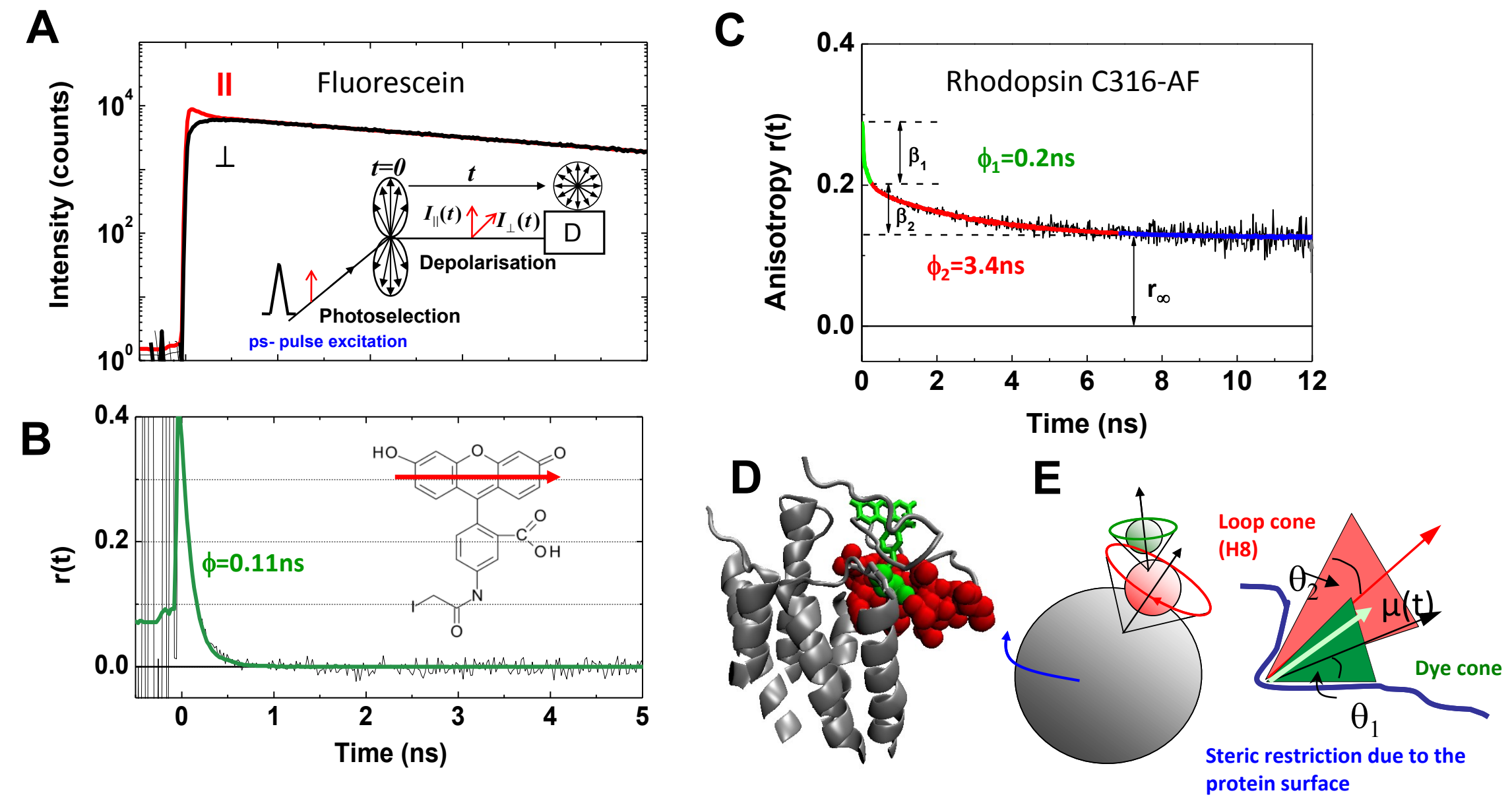

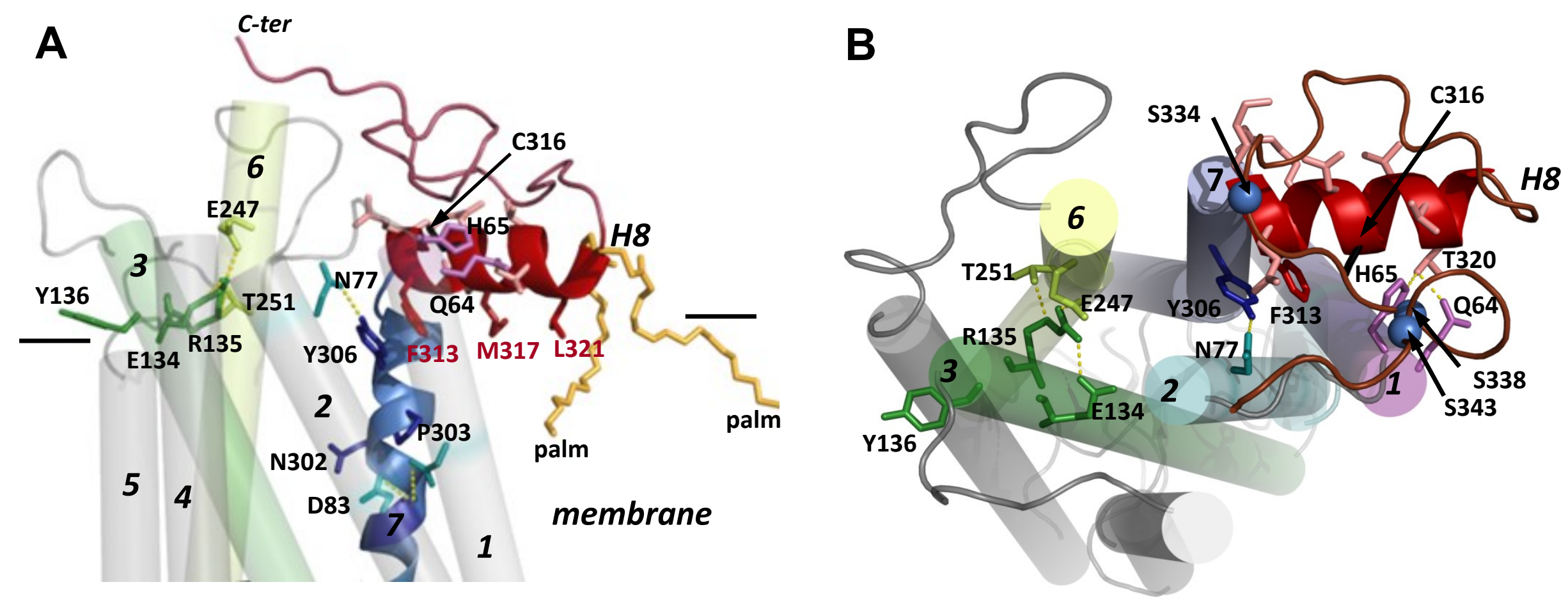

Figure 3 
Figure 4
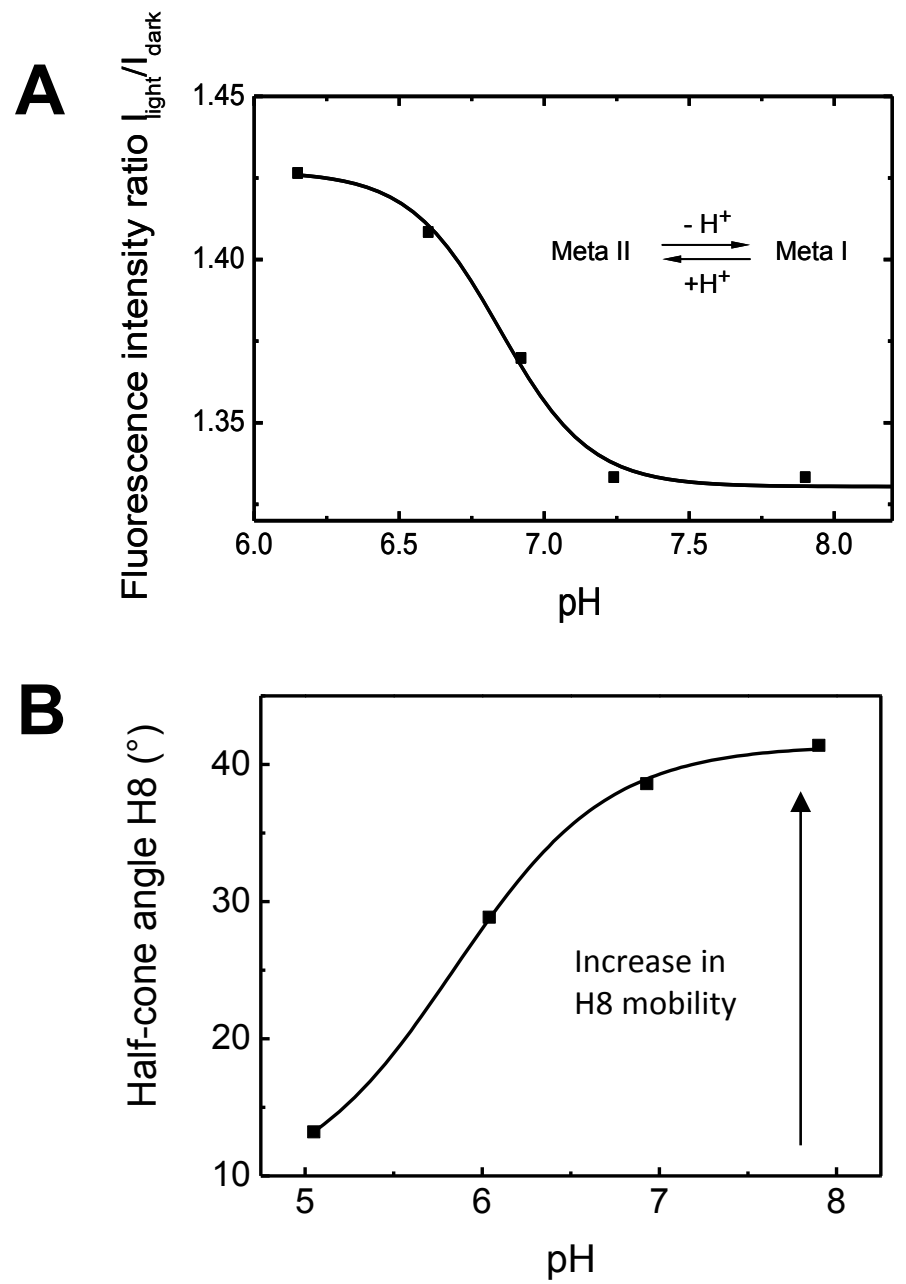
Figure 5

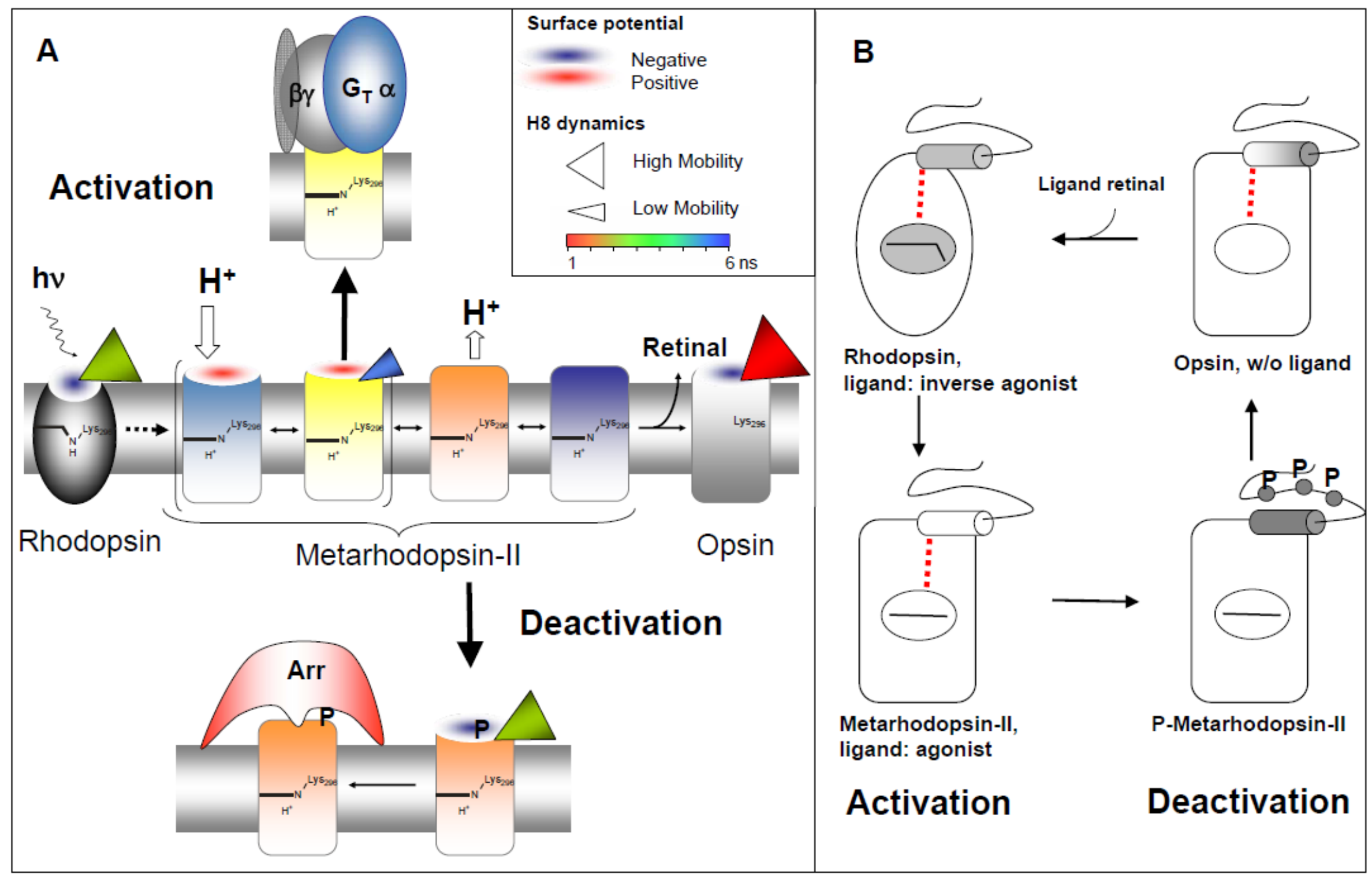


Figure 6

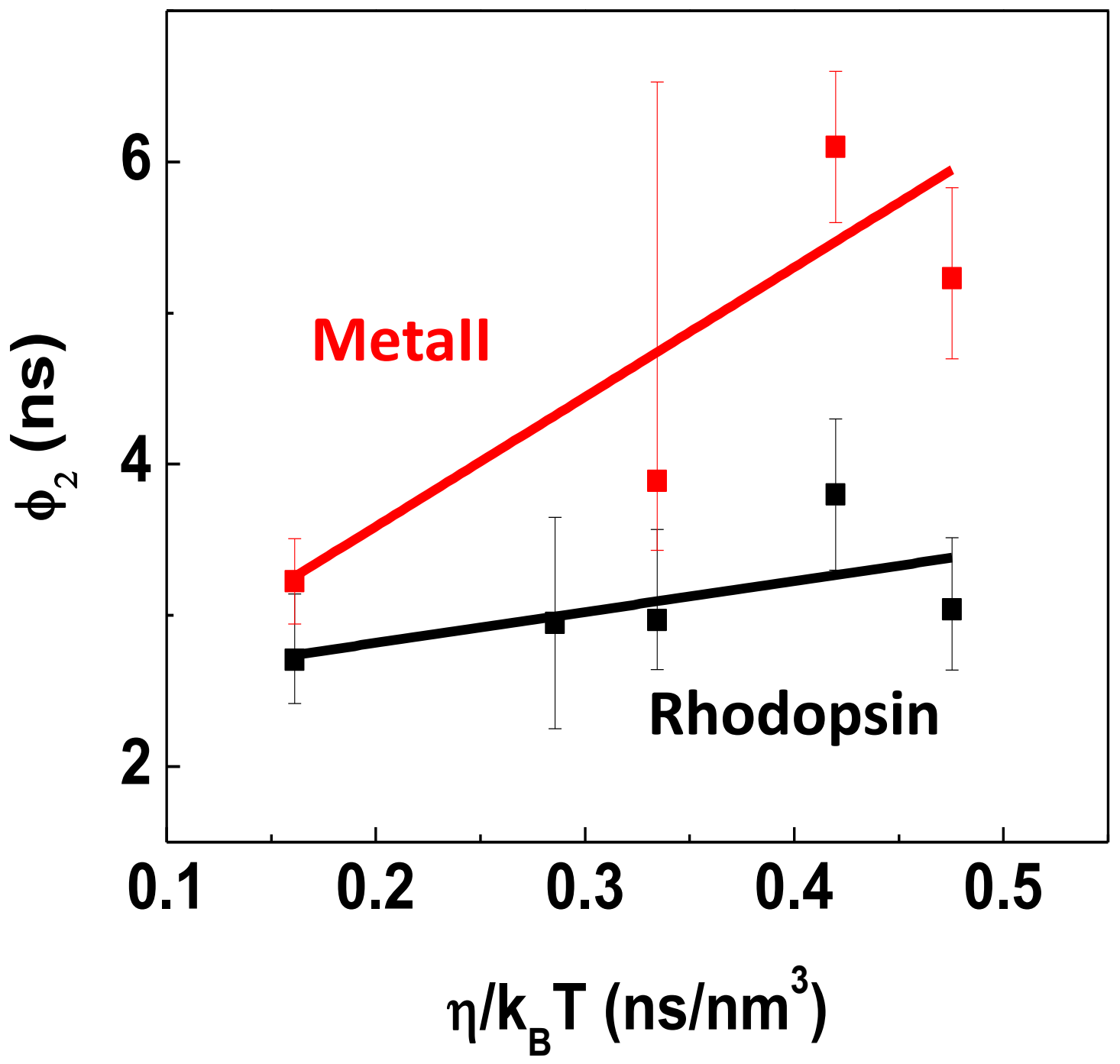


Figure 7
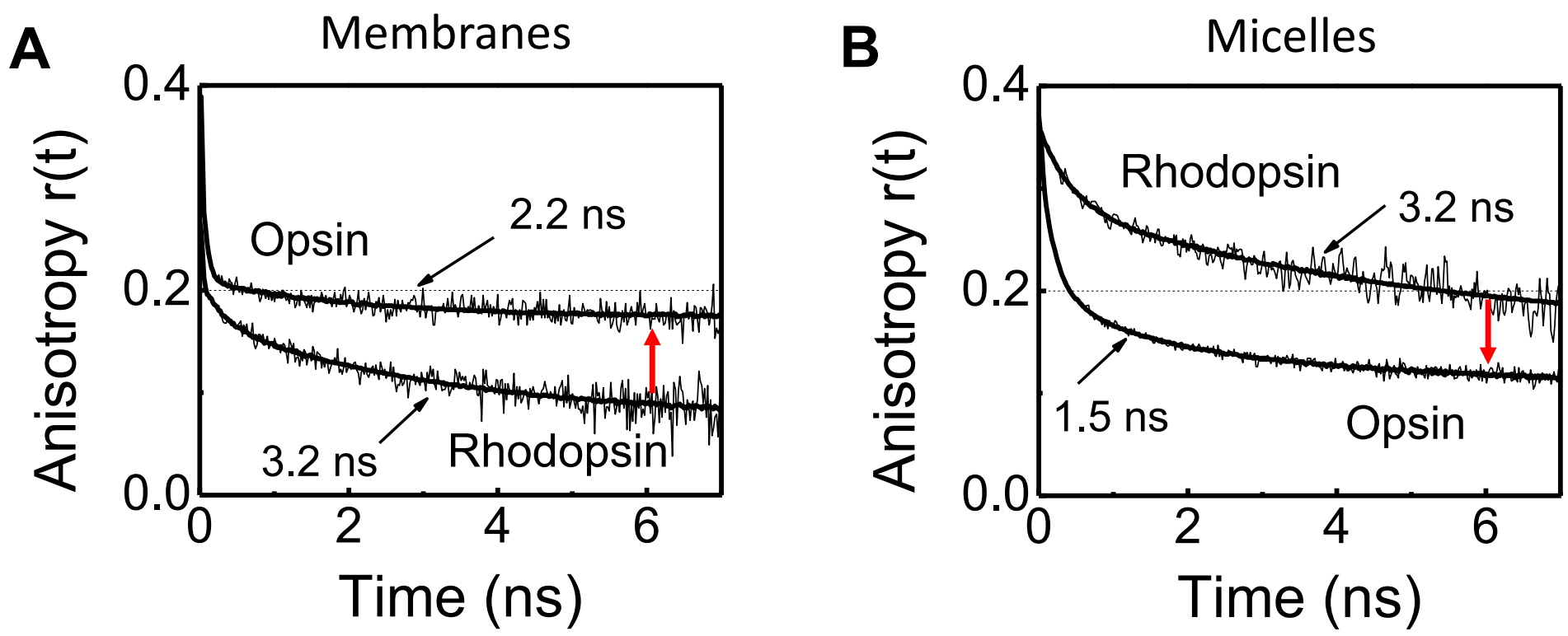
Figure 8
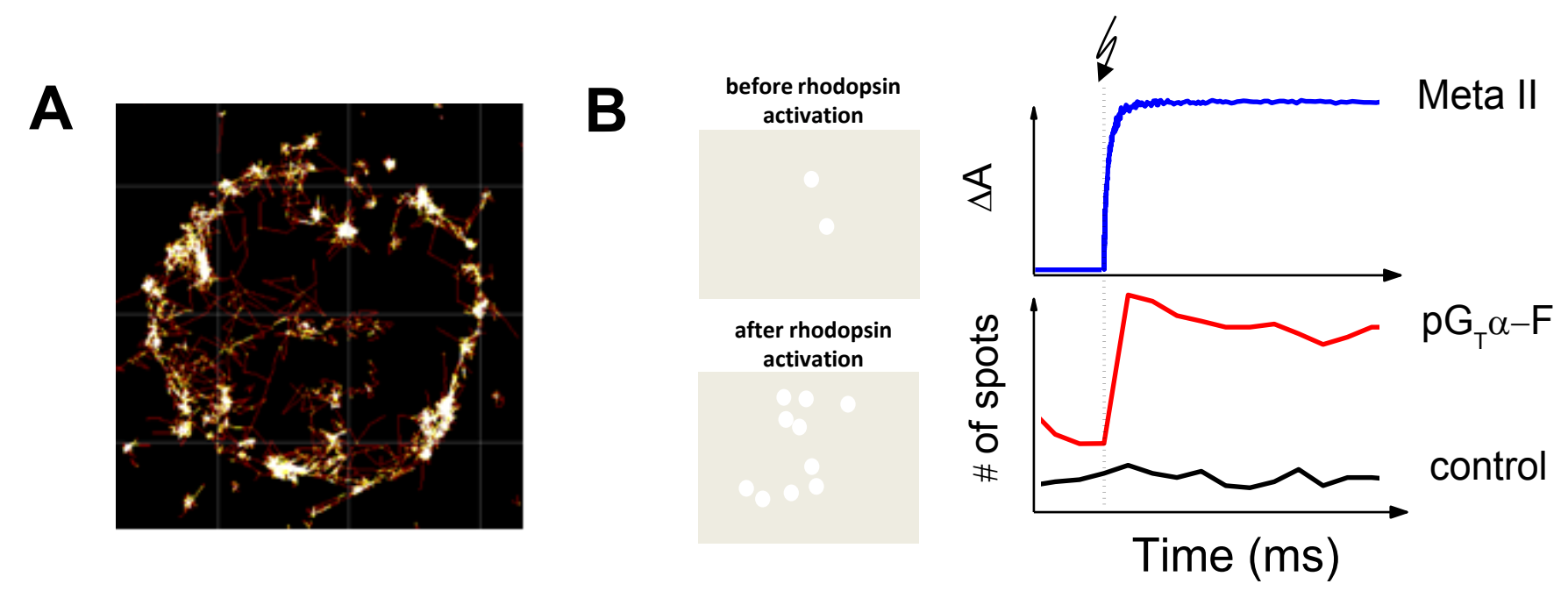